\title{
PAPER \\ Low-Profile Design of Metasurface Considering FSS Filtering Characteristics
}

Yuki KAWAKAMI $^{\dagger a)}, \begin{array}{r}\text { Student Member, Toshikazu HORI } \\ { }^{\dagger}\end{array}$, Fellow, Mitoshi FUJIMOTO ${ }^{\dagger}$,
Ryo YAMAGUCHI ${ }^{\dagger \dagger}$, Senior Members, and Keizo CHO

\begin{abstract}
SUMMARY This paper describes a metasurface designed utilizing either a Frequency Selective Surface (FSS) that has band-pass characteristics or one with band-rejection filtering characteristics in order to clarify the relationship between the filtering characteristics of the FSS and the Perfect Magnetic Conductor (PMC) characteristics of the metasurface. The effects of the filtering characteristics of the FSS on the PMC characteristics of the metasurface are described. Calculation results confirm that a low profile metasurface can be achieved using these FSSs. In addition, the effects of the size of the metasurface on the PMC characteristics of the surface are shown.

key words: metasurface, PMC, FSS, low-profile
\end{abstract}

\section{Introduction}

A Frequency Selective Surface (FSS) comprises narrowspaced metal patches, and has frequency-band-rejection or frequency-band-pass characteristics [1], [2]. It is known that a FSS with the ground plane has Perfect Magnetic Conductor (PMC) characteristics in a specific frequency band. This surface is referred to as a metasurface and it can control the phase of a wave that is reflected at the surface [3], [4]. A metasurface is applied to antennas [5]-[9], filters [10], and absorbers [11] to improve the performance of these devices. There are various purposes to apply metasurfaces to antennas, for example, suppressing the mutual coupling between antenna elements [5], [6], improving the gain of antennas [7], [8], and miniaturizing the antennas [9]. When the goal is to achieve a low profile antenna, a low profile metasurface is required.

Several design methods incorporating a metasurface have been proposed. One method is to employ equivalent circuit models [12]. A resonant cavity approach using ray theory was presented in [13] and [14]. In [15] and [16], optimization algorithms such as Genetic algorithms were used to design a metasurface. A number of metasurfaces have already been proposed by using these methods. However, the relationship between the filtering characteristics of the FSS and the PMC characteristics of the metasurface which is designed utilizing the FSS has not yet been clarified. The PMC characteristics of the metasurface change as the filter-

Manuscript received March 18, 2011.

Manuscript revised September 5, 2011.

$\dagger$ The authors are with the Graduate School of Engineering, University of Fukui, Fukui-shi, 910-8507 Japan.

${ }^{\dagger}$ The authors are with NTT DOCOMO, Inc., Yokosuka-shi, 239-8536 Japan.

a)E-mail: y.kawakami@m.ieice.org

DOI: $10.1587 /$ transcom.E95.B.477 ing characteristics of the FSS change. There are optimum filtering characteristics that are suitable for the design of the required metasurface.

In this paper, a low profile metasurface is designed by utilizing either a FSS that has band-pass characteristics or one that has band-rejection filtering characteristics. The design method uses an approach employing approximate ray theory because its logic is very simple. The theory provides a physical insight of the wave propagation phenomena by describing the total wave fields as a sum of different types of waves. It can directly calculate the characteristics of waves. The design method utilized in [13], [14] is expanded to design the metasurface by using the FSS with various filtering characteristics. The metasurface comprising the FSS which has the total transmission characteristics has already been designed. In this paper, the metasurface utilizing the FSS which has the partial reflection characteristics is designed. The effects of the filtering characteristics of the FSS on the PMC characteristics of the metasurface which is designed utilizing the FSS are described. Furthermore, reflection characteristics of a finite-size low-profile metasurface are clarified.

In Sect. 2, the metasurface configuration is described. A loop-slot or a loop type FSS are utilized to design the metasurface. In Sect. 3, a design method for the metasurface using ray theory is explained. In Sect. 4 , the effect of the filtering characteristics of the FSS on the PMC characteristics of the metasurface comprising the FSS is examined. In Sect. 5, reflection characteristics of a finite-size low-profile metasurface are described. In order to verify the obtained calculation results, the designed metasurface is fabricated. In Sect. 6, we present our conclusions.

\section{Configuration and Analysis Models of Metasurface}

Figure 1 shows the configuration of the metasurface and the analysis models for the FSS elements, FSS, and metasurface. Figure 1(a) indicates the configuration of a loop-slot type metasurface. As shown in Fig. 1(a), the metasurface comprises the FSS and the ground plane. Figure 1(b) indicates the shape of the FSS elements. As shown in Fig. 1(b), we consider two types of FSSs. One is a loop-slot type FSS which has band-pass characteristics, and the other is a loop type FSS which has band-rejection characteristics. In this paper, the loop-slot and loop type FSSs have complementary structures in each other. Here, the frequency $f_{\mathrm{BP}}$ is 


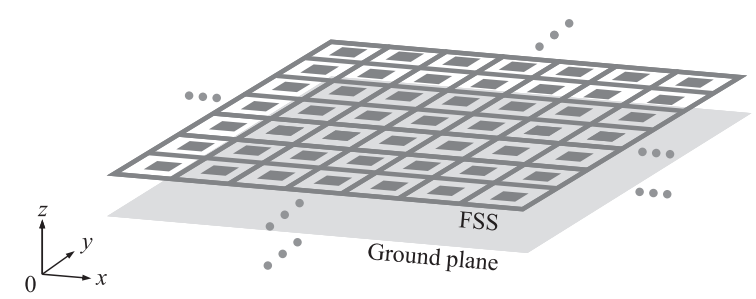

(a) Configuration of loop-slot type metasurface

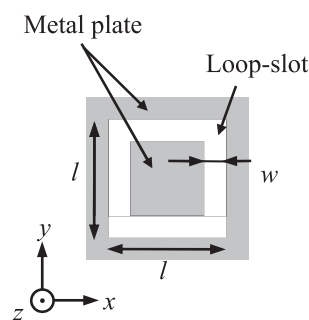

(i) Loop-slot type

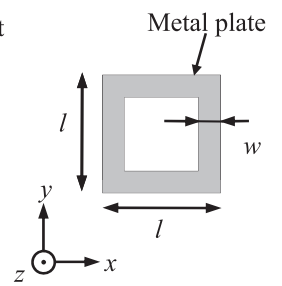

(ii) Loop type (b) Shape of FSS element

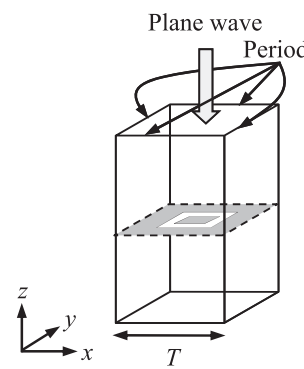

(c) FSS structure
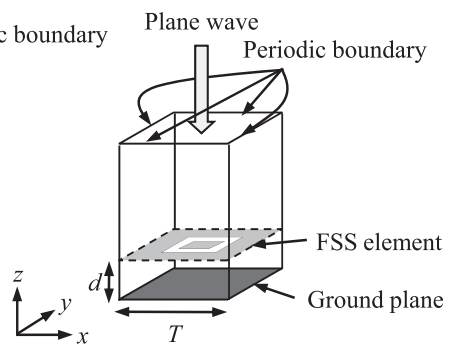

(d) Metasurface structure
Fig. 1 Configuration and analysis models.

defined as the frequency where the loop-slot type FSS has band-pass characteristics. The frequency $f_{\mathrm{BR}}$ is defined as the frequency where the loop type FSS has band-rejection characteristics. The reason for choosing these FSSs is described in Sect. 3. The difference in PMC characteristics of the metasurface that is obtained using these FSSs is discussed in Sect. 4. Figures 1(c) and 1(d) show the analysis models for FSS and metasurface structures in the case of the loop-slot type. Each FSS comprises FSS elements shown in Fig. 1(b) arranged on the xy-plane infinitely. The parameter $T$ is the period of arrangement, $w$ is the width of a loopslot/loop, $d$ is the distance between the FSS and the ground plane, and $l$ is the length of the circumference of a loopslot/loop. In order to analyze the infinite structures, the periodic boundary condition (PBC) is utilized in the FDTD analysis.

\section{Metasurface Design Method}

In this section, we discuss the design method for the metasurface comprising FSS at a frequency $f$ by using ray theory. Let $S_{11}$ and $S_{21}$ be the reflection coefficient and the insertion loss of the FSS at frequency $f$, respectively. When a plane wave propagates from the positive $z$ direction as shown in

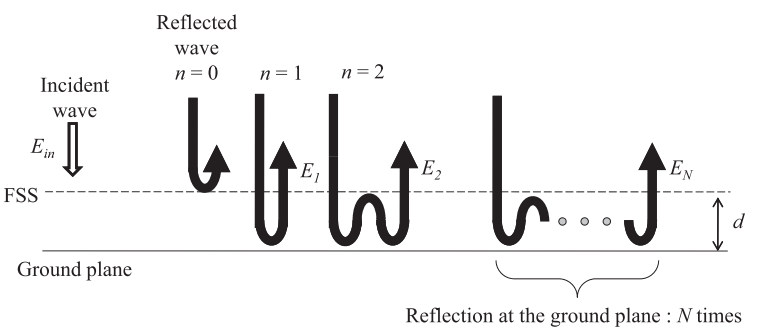

Fig. 2 Route of reflected wave at metasurface composed of FSS and ground plane.

Fig. 2 and arrives at the FSS, the amplitudes of the waves reflected by the FSS layer and penetrated through the FSS layer are the $\left|S_{11}\right|$ and $\left|S_{21}\right|$ times that of the incident wave. The penetrated wave is reflected at the ground plane and going back to the FSS. Then the reflection and penetration are repeated as shown in Fig. 2. Here, $n$ is the number of times that the wave is reflected at the surface of the ground plane. At $f_{\mathrm{BP}}$, all waves pass through the FSS layer, that is, only the reflected wave of $n=1$ is considered as the reflected wave. Conversely, only the wave of $n=0$ is considered at $f_{\mathrm{BR}}$ because all waves are reflected at the surface of the FSS. Here, $E_{\text {in }}$ is the electric field of the incident wave, $E_{\text {total }}$ is the electric field of the wave comprising all the reflected waves going back to the positive $\mathrm{z}$ direction which are reflect by or penetrate through the FSS, and $n$ is the number of times that the wave is reflected at the surface of the ground plane. In the case of $n=0,1$, and 2, the electric field of the reflected waves going (or propagating) back to the positive $z$ direction is represented as

$$
\begin{aligned}
& E_{0}=E_{i n}\left|\mathrm{~S}_{11}\right| e^{j \phi_{11}}, \\
& E_{1}=E_{i n}\left|\mathrm{~S}_{21}\right|^{2} e^{j\left(2 \phi_{21}+2 \phi_{\varepsilon}+\phi_{\text {ref }}\right)}, \\
& E_{2}=E_{i n}\left|\mathbf{S}_{21}\right|^{2}\left|\mathrm{~S}_{11}\right| e^{j\left(\phi_{11}+2 \phi_{21}+4 \phi_{\varepsilon}+2 \phi_{\text {ref }}\right)} .
\end{aligned}
$$

Here, $\phi_{11}, \phi_{21}, \phi_{\varepsilon}$, and $\phi_{\text {ref }}$ are the reflection phase of the FSS, the transmission phase of the FSS, the phase rotation between the FSS and the ground plane, and the reflection phase of the ground plane, respectively. The phase rotation $\phi_{\varepsilon}$ can be expressed as

$$
\phi_{\varepsilon}=\frac{2 \pi d}{\lambda} .
$$

The equations of the electric field of the reflected waves are a geometrical progression the first term for which is equal to $E_{1}$ and common ratio $r=\left|\mathrm{S}_{11}\right| \mathrm{e}^{\mathrm{j}\left(\phi_{11}+2 \phi_{\varepsilon}+\phi_{\text {ref }}\right)}$ when $n$ is greater than 1 . Therefore, electric field $E_{\text {total }}$ of the wave comprising all the reflected waves from $n=0$ to $N$ can be expressed as

$$
E_{\text {total }}=E_{0}+\sum_{n=1}^{N} E_{n}=E_{0}+\frac{E_{1}\left(1-r^{N}\right)}{1-r} .
$$

If we rearrange Eq. (5) with $N \rightarrow \infty$, the following equation is obtained

$$
E_{\text {total }}=E_{0}+\frac{E_{1}}{1-r} \text {. }
$$

When the amplitude and phase of the reflected wave $E_{\text {total }}$ 
is equal to that of the incident wave $E_{\mathrm{in}}$ at the FSS, this situation corresponds to when the wave is reflected at the surface without phase rotation. Namely the FSS with the ground plane has the PMC characteristics when the electric field $E_{\text {total }}$ satisfies Eq. (7).

$$
E_{\text {total }}=E_{\text {in }} .
$$

In this paper, we assume that $E_{i n}=1$. Then, the following equation is obtained by substituting Eq. (6) into Eq. (7).

$$
E_{0}+\frac{E_{1}}{1-r}=1 .
$$

The specific distance $d$ between the FSS and ground plane obtaining PMC characteristics can be derived by substituting Eq. (2), Eq. (4) and common ratio $r=\left|\mathrm{S}_{11}\right| \mathrm{e}^{\mathrm{j}\left(\phi_{11}+2 \phi_{\varepsilon}+\phi_{\text {ref }}\right)}$ into Eq. (8).

$$
d=j \frac{\lambda}{4 \pi} \log \left[\frac{1-E_{0}}{e^{j \phi_{r e f}}\left\{\left|\mathrm{~S}_{21}\right|^{2} e^{j 2 \phi_{21}}+\left(1-E_{0}\right) E_{0}\right\}}\right] .
$$

In this paper, we use Eq. (9) to design the metasurface.

As shown in this section, PMC characteristics of the metasurface are obtained by synthesizing the waves that have different routes. The route means that each wave is reflected at the different number of times by the ground plane. We need to utilize the FSS that has both of reflection and penetration characteristics. Hence a loop-slot and loop type FSS are utilized in this paper. These FSSs have the both characteristics at the frequency except $f_{\mathrm{BP}}$ and $f_{\mathrm{BR}}$.

\section{Design of Metasurface Comprising FSS}

\subsection{FSS Filtering Characteristics}

As described in Sect. 3, the filtering characteristics of the FSS must be determined to design the metasurface by using Eq. (7). To define the filtering characteristics, the transmission and reflection characteristics of the FSS when the plane wave comes from the positive $z$ direction are calculated by using FDTD method. The model presented in Fig. 1(c) is utilized.

Figure 3 shows the filtering characteristics of the loopslot and loop type FSSs. The parameters $l=0.01 \lambda_{\mathrm{BP}}, w$ $=0.25 \lambda_{\mathrm{BP}}$, and $T=0.26 \lambda_{\mathrm{BP}}$. Here, $\lambda_{\mathrm{BP}}$ is defined as the wavelength at the frequency $f_{\mathrm{BP}}$. Figure 3(a) shows the amplitude of the S-parameters, and Fig. 3(b) shows the phase of the S-parameters. The black lines and the gray lines indicate the results of the loop-slot and the loop type FSSs, and the solid line and the dashed line represent the characteristics of $S_{11}$ and $S_{21}$, respectively. The vertical axis and horizontal axis show the amplitude of S-parameters and the frequency that was normalized by $f_{\mathrm{BP}}$.

As can be seen in Fig. 3(a), the loop-slot type FSS with the structural parameters described in above operates as a band-pass filter. It is also seen that the loop type FSS, which is complementary structure of the loop-slot type FSS, operates as a band-rejection filter around the frequency of 0.9

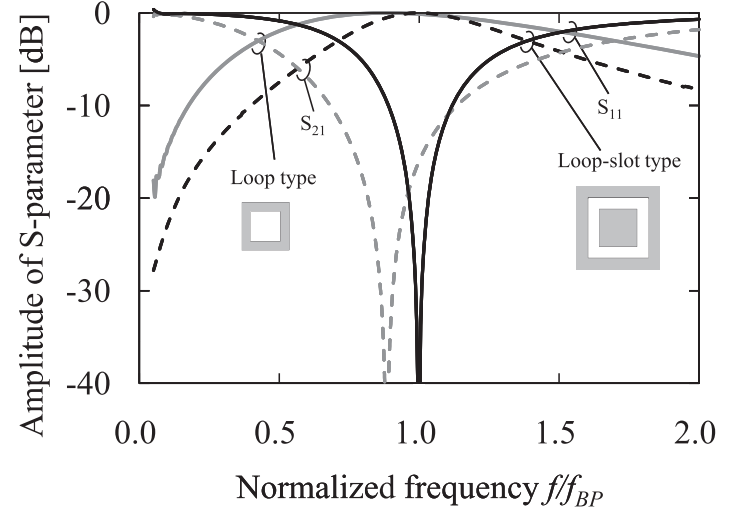

(a) Amplitude of S-parameter

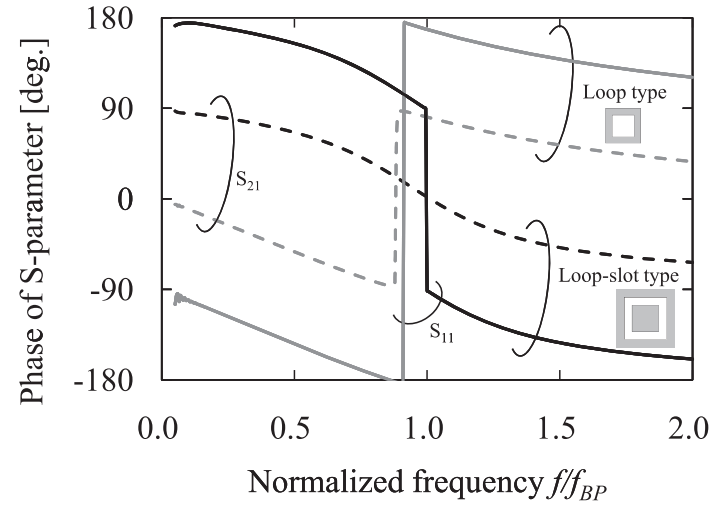

(b) Phase of S-parameter

Fig.3 Filtering characteristics of loop-slot/Loop Type FSS

$f_{\mathrm{BP}}$. Thus we can confirm that both types have almost inverse filtering characteristics. In addition, we can see a discontinuity in the phase characteristics at the frequency $f_{\mathrm{BP}}$ and $0.9 f_{\mathrm{BP}}$ for loop-slot and loop type FSSs, respectively.

\subsection{Transition of Synthesized Electric Field, $E_{\text {total }}$}

As explained in previous section, only the reflected wave of $n=1$ should be considered as the reflected wave at $f_{\mathrm{BP}}$. Similarly, only the reflected wave of $n=0$ should be considered as the reflected wave at $f_{\mathrm{BR}}$. At other frequencies, there are various reflected waves with different amplitudes, phases, and routes. Thus all these waves should be considered at these frequencies. In this section, we discuss the reflected waves at frequencies other than $f_{\mathrm{BP}}$ and $f_{\mathrm{BR}}$.

Figure 4 shows the transition of the synthesized electric field, $E_{\text {total }}$, at $0.5 f_{\text {BP. }}$. Figures 4(a) and 4(b) show the cases for the loop-slot and loop type FSSs, respectively. This figure 4(a) shows the cases when the distance $d$ between the FSS layer and the ground plane of $0.460 \lambda_{0.5 f_{\mathrm{BP}}}, 0.463$ $\lambda_{0.5 f_{\mathrm{BP}}}, 0.465 \lambda_{0.5 f_{\mathrm{BP}}}$, and $0.468 \lambda_{0.5 f_{\mathrm{BP}}}$. In Fig. 4(b), the cases for the distance $d$ of $0.051 \lambda_{0.5 f_{\mathrm{BP}}}, 0.056 \lambda_{0.5 f_{\mathrm{BP}}}, 0.061 \lambda_{0.5 f_{\mathrm{BP}}}$, and $0.066 \lambda_{0.5 f_{\mathrm{BP}}}$ is indicated. Here, $\lambda_{0.5 f_{\mathrm{BP}}}$ is the wavelength at the frequency $0.5 f_{\mathrm{BP}}$. Only in the case of specific distance $d=0.468 \lambda_{0.5 f_{\mathrm{BP}}}$ or $0.051 \lambda_{0.5 f_{\mathrm{BP}}}$, the process in which 


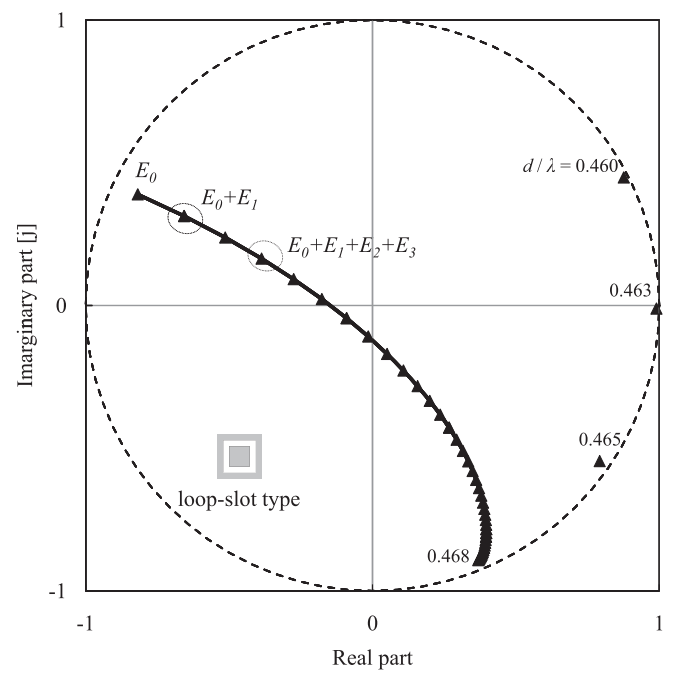

(a) Loop-slot type

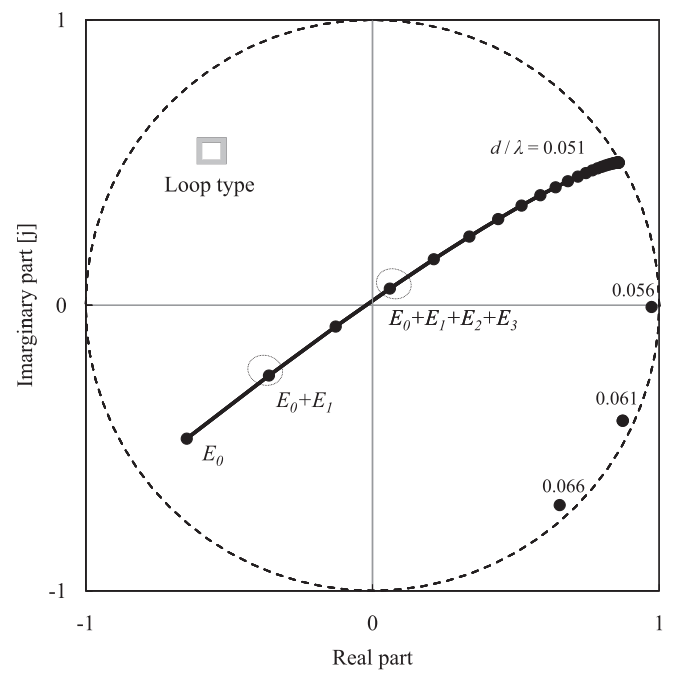

(b) Loop type

Fig. 4 Transition of $E_{\text {total }}\left(f=0.5 f_{\mathrm{BP}}, N=50\right)$.

the reflected waves are synthesized is plotted. We consider reflected waves from $n=0$ to 50 , that is, the transition of $E_{\text {total }}$ comprises $E_{0}$ to $E_{50}$ and is drawn in Fig. 4. In the case of other distances $d$, only the results are plotted.

As shown in Fig. 4, it is represented that the electric field $E_{\text {total }}$ converges at a certain value as the number $n$ of reflected waves is increased in both cases. It is also shown that the converged electric field changes as distance $d$ changes. The absolute value of the converged electric field $E_{\text {total }}$ is nearly equal to 1 regardless of distance $d$. For the loop-slot type FSS, when distance $d=0.463 \lambda_{0.5 f_{\mathrm{BP}}}$, the imaginary part of the electric field $E_{\text {total }}$ equals 0 and the real part of $E_{\text {total }}$ is greater than 0 . Accordingly, the metasurface, which operates at the frequency $0.5 f_{\mathrm{BP}}$ can be designed by setting distance $d=0.463 \lambda_{0.5 f_{\mathrm{BP}}}$. Similarly, for the loop type FSS, the metasurface that operates at the frequency $0.5 f_{\mathrm{BP}}$ can be designed by setting distance $d=0.056 \lambda$. Consequently, it is clear that a thinner metasurface can be designed by using a

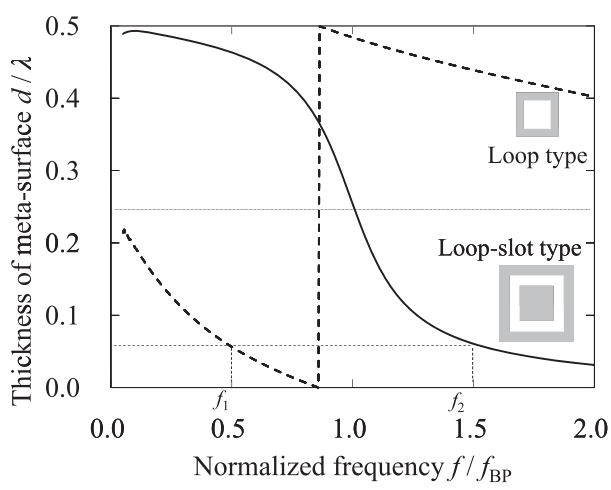

(a) Thickness

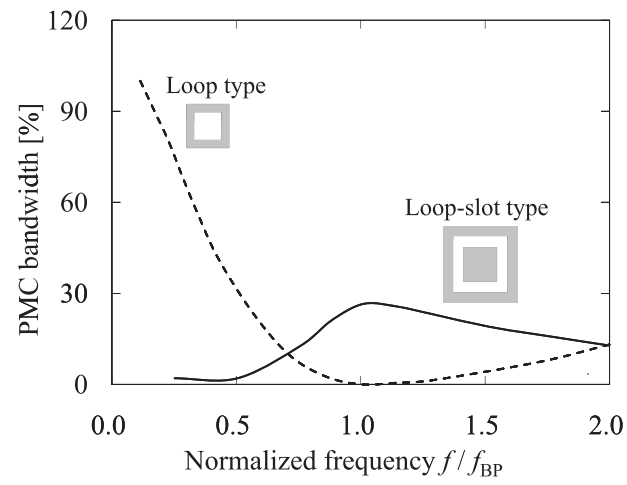

(b) PMC bandwidth

Fig. 5 Thickness and PMC bandwidth of designed metasurface.

loop type FSS at the frequency $0.5 f_{\mathrm{BP}}$.

\subsection{Thickness and PMC Bandwidth of Metasurface}

Figure 5 indicates the thickness and the PMC bandwidth of the designed metasurface. Here, the thickness means the distance between the FSS layer and the ground plane when PMC characteristics can be realized at each normalized frequency. Figure 5(a) shows the thickness, and Fig. 5(b) shows the PMC bandwidth. Here, the PMC bandwidth is defined as the frequency band within the reflection phase of \pm 90 degrees. In both figures, the solid line and dashed line represent the cases for the loop-slot and loop type FSSs, respectively.

For the loop-slot type FSS, the thickness of the metasurface becomes thinner as the design frequency for metasurface becomes higher. On the other hand, for the loop type FSS, the thickness of the metasurface at the frequency less than $0.9 f_{\mathrm{BP}}$ tends to be thinner than that obtained at the frequency more than $0.9 f_{\mathrm{BP}}$. Consequently, the thickness of the metasurface for the loop type FSS is thinner (thicker) than that for the loop-slot type at a low (high) frequency. In the case of the loop-slot type FSS, we can achieve a metasurface with the thickness of $0.05 \lambda$ at the frequency $1.5 f_{\mathrm{BP}}$. On the other hand, for the loop type FSS, a metasurface with the thickness of $0.05 \lambda$ can be obtained at the frequency 0.5 $f_{\mathrm{BP}}$. 
It is shown in Fig. 5(b) that the PMC bandwidth of the metasurface changes as the frequency changes. In addition, we find that there is a peak in the PMC bandwidth in the case of the loop-slot type FSS. The peak is obtained at the frequency $1.0 f_{\mathrm{BP}}$. The frequency corresponds to the frequency where the amplitude of $S_{21}$ of the FSS nearly equals $0 \mathrm{~dB}$. For the loop-slot type FSS, the maximum PMC bandwidth of the metasurface is $26.5 \%$. On the other hand, we can achieve a thinner metasurface using the loop type FSS. The metasurface with a $100 \%$ PMC bandwidth is obtained at the frequency $0.1 f_{\mathrm{BP}}$.

To obtain a thin metasurface, we have to design it at lower (higher) frequency by using loop (loop-slot) type FSS. To achieve a wideband metasurfacce, we need to design it at the frequency $1.0 f_{\mathrm{BP}}$ for the loop-slot type FSS. In the case of the loop type FSS, we need the frequency to be $0.1 f_{\mathrm{BP}}$.

\section{PMC Characteristics of Finite Size Low-Profile Metasurface}

\subsection{Effects of Number of Elements on PMC Characteris- tics}

In the previous section, we assume that the metasuface has infinite size. However, an actual low-profile metasurface would have a finite structure. Therefore, the metasurface must be re-designed taking account of a finite size.

Figure 6 shows the reflection phase at the surface of the metasurface (phase of $E_{\text {total }}$ ). Figures 6(a) and 6(b) show the cases for the loop type and loop-slot type FSSs, respectively. Here, we consider the low-profile metasurface with the thickness $d$ of $0.05 \lambda$. From Fig. 5(a), the design frequency $f_{1}$ with PMC characteristics is $0.50 f_{\mathrm{BP}}$ for a loop type, and the frequency $f_{2}$ is $1.46 f_{\mathrm{BP}}$ for a loop-slot type. In Fig. 6, the solid line, dashed line, and dotted line indicate an infinite structure, 7 by 7 element structure, and 5 by 5 element structure, respectively.

As shown in Fig. 6, the frequency $f_{\mathrm{PMC}}$ where the metasurface has PMC characteristics becomes lower as the number of element is increased. When the number of elements is 7 by 7 , the frequencies with PMC characteristics are 0.57 $f_{\mathrm{BP}}$ for the loop type FSS and $1.46 f_{\mathrm{BP}}$ for the loop-slot type FSS. The error $\Delta f_{\mathrm{PMC}}$ of the design frequency is $10 \%$ in the case of loop type FSS. That for loop-slot type FSS is $0.3 \%$. Here, the error $\Delta f_{\mathrm{PMC}}$ is defined as the relative error between the frequency $f_{\mathrm{PMC}}$ for infinite size metasurface and that for finite size. As compare the cases for the loop type and the loop-slot type FSSs, it is found that the error $\Delta f_{\mathrm{PMC}}$ of them is different although the number of their elements is same.

The period $T$ of arrangement for the loop type FSS and that for the loop-slot type FSS is same in this paper. However, the design frequency where the metasurface is designed by using these FSSs is different. Thus the normalized size, which is normalized by the wavelength at the designed frequency, of these metasurfaces with 7 by 7 elements is different. Accordingly, the effects of the normalized size of metasurfaces on the error $\Delta f_{\mathrm{PMC}}$ of them should be consid-

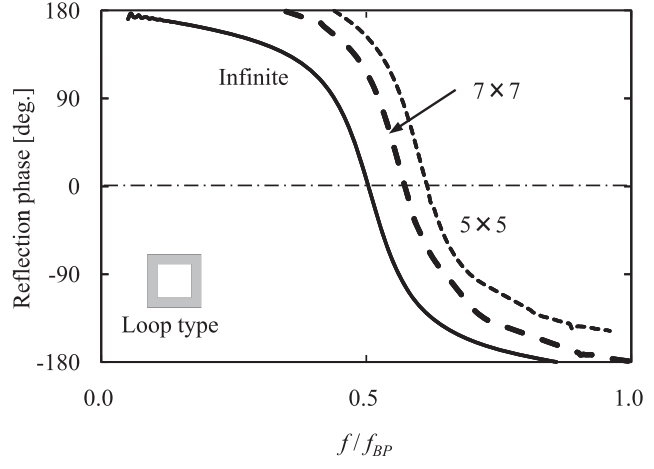

(a) Loop type

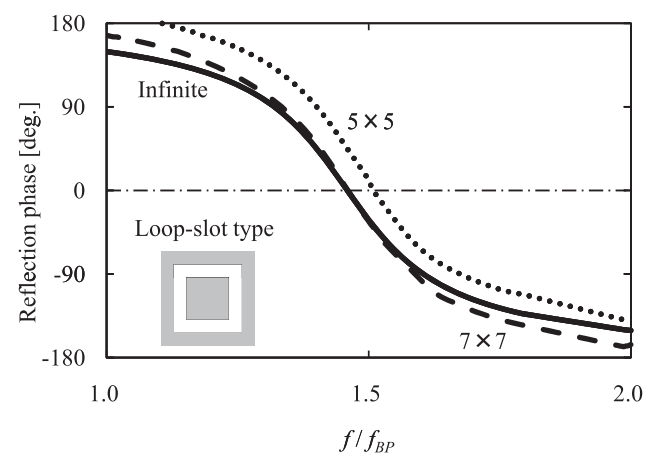

(b) Loop-slot type

Fig. 6 Reflection phase at the surface of low-profile metasurface.

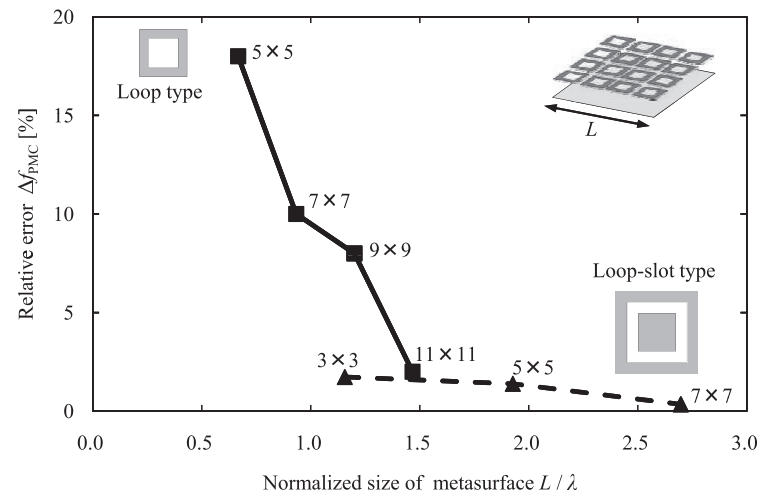

Fig. 7 Relative error of the designed frequency of finite metasurface.

ered. Figure 7 shows the relationship between the normalized size of metasurfaces and the error $\Delta f_{\mathrm{PMC}}$ of them. The vertical axis and horizontal axis show the error $\Delta f_{\mathrm{PMC}}$ of the finite size metasurface and the normalized length $L / \lambda$ of the circumference of the metasurface. In Fig. 7, the solid line and dashed line indicate the case of loop type and loop-slot type FSSs, respectively. As shown in Fig. 7, the error $\Delta f_{\mathrm{PMC}}$ becomes smaller as the normalized size of the metasurface is increased in both cases. It is also found that the error $\Delta f_{\mathrm{PMC}}$ can be suppressed to $2 \%$ or less by setting the size of the metasurface $L \geq 1.5 \lambda$. Consequently, the number of the elements should be decided so that the length $L$ of the finite size metasurface becomes $1.5 \lambda$ or more. 


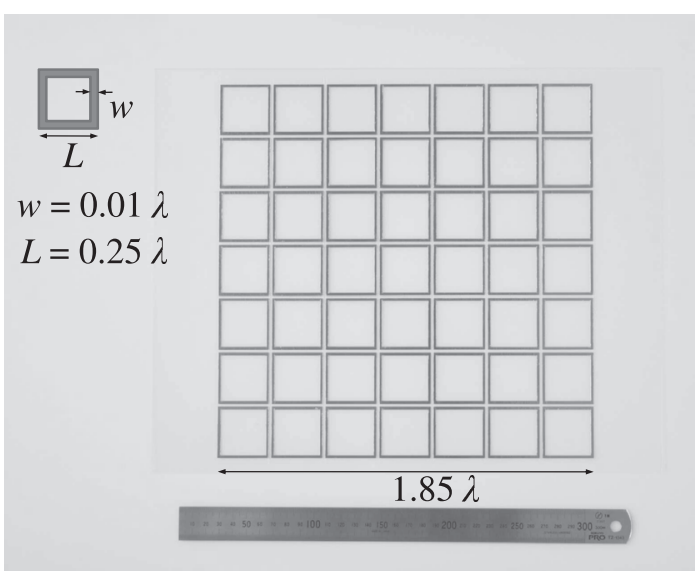

(a) Fabricated metasurface

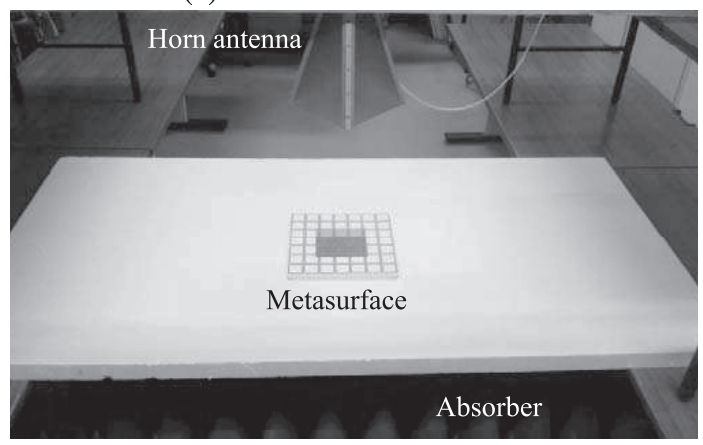

(b) Experimental setup

Fig. 8 Reflection phase at the surface of low-profile metasurface.

\subsection{Verification Through Experiment}

In order to verify the above-mentioned calculated results, the loop type metasurface with 7 by 7 element was fabricated.

Figure 8 shows a photograph of the experimental configuration. Figures 8(a) and 8(b) show a photograph of the fabricated metasurface and experimental environment. The FSS elements are constructed of copper. The size of elements is shown in Fig. 8(b) and that of the ground plane is 7 $T \times 7 T$. The thickness of the metasurface is set to $0.1 \lambda$. As shown in Fig. 8(b), a horn antenna is utilized to generate an incident plane wave in the experiment. When the reflection phase of the metasurface is measured, a flat metal plane of the same size with the metasurface is utilized as a reference.

Figure 9 indicates the calculated and experimental reflection phase of the finite metasurface. The solid line represents the experimental results and the dashed line represents the calculated results. As shown in Fig. 9, the calculated results almost agree with the experimental results. That is why we conclude that the calculate results are appropriate. In the case of the calculation, the frequency where the metasurface has PMC characteristics, $f_{\mathrm{PMC}}$, is $0.46 f_{\mathrm{BP}}$. On the other hand, in the experiment, the frequency $f_{\mathrm{PMC}}$ equals $0.48 f_{\mathrm{BP}}$.

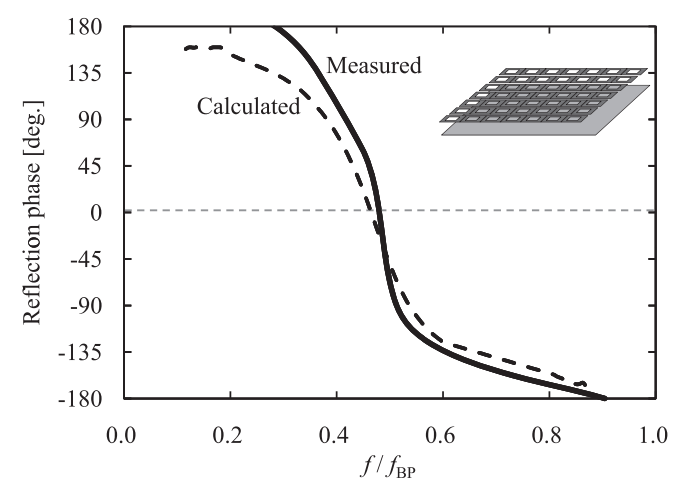

Fig. 9 Reflection phase at the surface of low-profile metasurface.

\section{Conclusion}

A novel design method based on the ray theory was proposed to design the metasurface by using the FSS which has partial reflection characteristics. The metasurface was designed using a loop-slot or loop type FSS. Based on the simulation results, it was clarified that a low-profile metasurface could be achieved by using these FSSs. It had a low profile structure with the thickness of $1 / 10$ wavelength. Furthermore, the effects of the size of the metasurface on the frequency where the surface has PMC characteristics were shown. It was clarified that the number of the elements should be decided so that the length of the circumference of the metasurface becomes $1.5 \lambda$ or more. Finally, the validity of the design method was presented by the experimental results.

\section{References}

[1] B.A. Munk, Frequency Selective Surfaces: Theory and Design, John Wiley \& Sons, New York, NJ, 2000.

[2] E.A. Parker, R.J. Langley, R. Cahill, and J.C. Vardaxoglou, "Frequency selective surfaces," IEE Proc., ICAP'83, vol.1, pp.459-463, Norwich, UK, April 1983.

[3] J.C. Vardaxoglou, A. Chauraya, R.D. Seager, N. Riauka, G.K. Palikaras, and P deMaagt, "Towards metasurfaces for wideband systems,” METAMATERIALS'2009, pp.141-143, London, Sept. 2009.

[4] E. Saenz, et al., "Coupling reduction between dipole antenna elements by using a planar meta-surface," IEEE Trans. Antennas Propag., vol.57, no.2, pp.383-394, Feb. 2009.

[5] F. Yang and Y. Rahmat-Samii, "Mutual coupling reduction of microstrip antennas using electromagnetic band-gap structure," IEEE Antennas Propagation Society International Symposium, vol.2, pp.478-481, July 2001.

[6] Y. Kawakami, T. Hori, M. Fujimoto, R. Yamaguchi, and K. Cho, "Mutual coupling suppression property by three dimensionally arranged EBG structures,” Proc. ISAP2010, Macao, China, Nov. 2010.

[7] H.N. Liu, H.L. Su, K.H. Lin, C.Y. Wu, C.L. Tang, and S.H. Yeh, "Design of antenna radome composed of metamaterials for high gain," IEEE Antennas and Propagation Society International Symposium, pp.19-22, July 2006.

[8] Y. Kawakami, T. Hori, M. Fujimoto, R. Yamaguchi, and K. Cho, "Radiation properties of rectangular microstrip antenna located by EBG structure,” Proc. ISAP2007, Niigata, Japan, Aug. 2007.

[9] F. Yang and Y. Rahmat-Samii, "Reflection phase characterizations of the EBG ground plane for low profile wire antenna applications," 
IEEE Trans. Antennas Propag., vol.51, no.10, pp.2691-2703, Oct. 2003.

[10] V. Radisic, Y. Qian, R. Coccioli, and T. Itoh, "Novel 2-D photonic bandgap structure for microstrip lines," IEEE Microw. Guided Wave Lett., vol.8, no.2, pp.69-71, Feb. 1998.

[11] A.K. Zadeh and A. Karlsson, "Capacitive circuit method for fast and efficient design of wideband radar absorbers," IEEE Trans. Antennas Propag., vol.57, no.8, pp.2307-2314, Aug. 2009.

[12] N. Engheta, "Thin absorbing screens using metamaterial surfaces," IEEE Antennas and Propagation Society International Symposium, vol.2, pp.392-395, Aug. 2002.

[13] A.P. Feresidis, G. Goussetis, and J.C. Vardaxoglou, "Metallodielectric Arrays without vias as Artificial Magnetic Conductors and electromagnetic Band Gap Surfaces," IEEE AP-S Int. Symp., vol.2, pp.1159-1162, June 2004.

[14] A.P. Feresidis, G. Goussetis, S. Wang and John (Yiannis) C. Vardaxoglou, "Artificial magnetic conductor surfaces and their application to low-profile high-gain planar antennas," IEEE Trans. Antennas Propag., vol.53, no.1, pp.209-215, Jan. 2005.

[15] D.J. Kern, D.H. Werner, A. Monorchio, L. Lanuzza, and M.J. Wilhelm, "The design synthesis of multiband artificial magnetic conductors using high impedance frequency selective surfaces," IEEE Trans. Antennas Propag., vol.53, no.1, pp.8-17, Jan. 2005.

[16] A. Fallahi, M. Mishrikey, C. Hafner, and R. Vahldieck, "Efficient procedures for the optimization of frequency selective surfaces," IEEE Trans. Antennas Propag., vol.56, no.5, pp.1340-1349, May 2008.

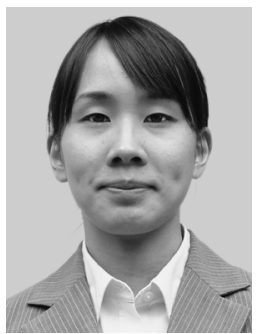

Yuki Kawakami was born in Fukui Prefecture, Japan, on March 27, 1985. she recieved the B.S., M.S. degrees from University of Fukui, Japan, in 2007 and 2009. She is currently studying toward the Dr. Eng. degree at the Graduate School of Engineering, the University of Fukui. Her research interests include metamaterials such as EBG structures. She received the Student Award and the Young Engineers Award from the Technical Committee on AP of the IEICE of Japan in 2007 and 2010. She received the Young Engineers Award from the IEICE of Japan in 2009.

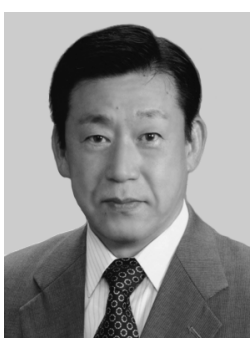

Toshikazu Hori received his B.E., M.E. and Dr. Eng. degrees from Kanazawa University, Japan, in 1974, 1976 and 1993, respectively. In 1976, he joined the Electrical Communications Laboratories, Nippon Telegraph and Telephone Public Corporation (now Nippon Telegraph and Telephone Corporation, NTT). Since then, he has been engaged in the research and development of antennas for satellite, cellular and microcellular mobile, and advanced wireless communication systems. In 2001, he moved to the University of Fukui, and is currently a Professor at the Graduate School of Engineering. His current research interests lie in the area of antennas and propagation for wireless broadband systems, especially, broadband antennas and adaptive antennas. Dr. Hori is a senior member of the IEEE, and a member of the ITE, Japan. He served as the Senior Editor of the Transactions of the IEICE on Communications from 2001 to 2003, the Vice-Chair of ISAP2004 Steering Committee, the Chair of ISAP2007 Technical Program Committee, and the Vice-Chair and the Chair of the Technical Committee on Antennas and Propagation (TC-AP) of IEICE from 2005 to 2009, respectively. He was also the Vice-Chair and the Chair of the IEEE AP-S Japan Chapter from 1999 to 2000, and the Vice-Chair and the Chair of the IEEE AP-S Nagoya Chapter from 2007 to 2010. He is now the Advisory Committee Member of the TC-AP of IEICE.

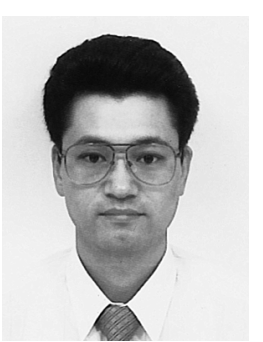

Mitoshi Fujimoto was born in Kagoshima, Japan, on June 20, 1964. In 1985, he joined the Toyota Central Research \& Development Laboratories, Inc. He received his B.S. degree in electronic engineering from Nagoya Institute of Technology, Japan, in 1989, his M.S. degree in electrical and computer engineering from the Institute in 1991, and a Doctorate in 2000, respectively. In 2003, he assumed a position at the University of Fukui, and is currently an Associate Professor there. His present interest lies in the area of adaptive arrays, terrestrial digital broadcasting, DOA estimation, MIMO technology, and UWB communications. He is currently an editor of the IEICE Transactions on Communications. He is a visiting researcher to the Advanced Telecommunications Research Institute International (ATR) and is member of the IEEE. He received the Young Engineer Award from the IEEE AP-S Tokyo chapter in 1992 and Distinguished Service Award from the IEICE Communications Society in 2005.

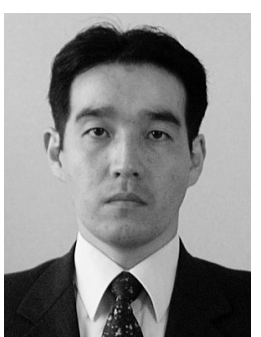

Ryo Yamaguchi received B.E. degree from Kyushu University in 1988, and M.S. and Dr. Eng. Degrees from Tohoku University in 1990 and 1994, respectively. He joined NTT Mobile Communications Network, Inc. in 1994 $\mathrm{He}$ is in NTT DOCOMO, INC. currently. He was engaged in studies on antennas and EMC for mobile communications. Dr. Yamaguchi received the Young Engineer Award from IEICE of Japan in 1995, and the Paper Award from IEICE in 2009. He is a member of IEEE.

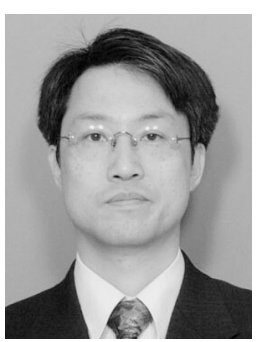

Keizo Cho received his B.E. degree from Yokohama National University, Japan in 1986 and his M.E. and Dr.Eng. degrees from Tokyo Institute of Technology, Japan, in 1988 and 2002, respectively. He is now Leader of the Antenna Project of the Research Laboratories, NTT DOCOMO, INC., Kanagawa, Japan. He has been engaged in the research and development of antennas for mobile communication systems. He served as the Secretary of Technical Committee on Antennas and Propagation of IEICE from 2003 to 2005, the Associate Editor of the Transaction of the IEICE Communications (Japanese edition) from 2005 to 2009. He is now the Associate Editor of the Transaction of the IEICE Communications (English edition) from 2010 and the Chair of the IEEE AP-S Japan Chapter. He received the Young Researchers' Award and Best Paper Award from the IEICE in 1995 and 2009, respectively. He is a senior member of the IEEE. 